\title{
Viral load as a risk factor of reverse transcriptase inhibitor drug resistance mutation in antiretroviral- treated people living with HIV/AIDS
}

\author{
Hotma Martogi Lorensi Hutapea ${ }^{1}$, Tri Nury Kridaningsih ${ }^{1}$, Khoirul Huda Prasetyo ${ }^{1}$, \\ and Milton Boaheng Antwi ${ }^{2}$
}

\section{ABSTRACT}

\section{BACKGROUND}

The human immunodeficiency virus type 1 (HIV-1) is a major contagion faced by the population of Indonesia. The success of antiretroviral treatment (ART) is threatened by the emergence of drug resistance mutations (DRM). The aim of this study was to determine the association between CD4 count, CD4 count changes, viral load, adherence to therapy, and therapy history in the presence of DRM in people living with HIV/AIDS (PLWHA).

\section{METHODS}

This was a cross-sectional study involving 269 adults who underwent antiretroviral (ARV) therapy for at least 6 months. The frequencies of DRM and polymorphisms were measured by partial amplification of the reverse transcriptase (RT) gene using RT-nested PCR on samples with viral loads of $>1000$ copies/mL. Sequencing was performed using the Sanger method, and edited by BioEdit. The edited sequences were submitted to http:// hivdb.stanford.edu for DRM determination. Respondents' medical data, CD4 count, viral load, and DRM were analyzed by simple and multiple logistic regression.

\section{RESULTS}

The multiple logistic regression analysis showed a significant association of CD4 count $(\mathrm{aOR}=12.47 ; 95 \% \mathrm{CI}: 1.45-107.39 ; \mathrm{p}=0.023)$ and viral load at the time of study $(\mathrm{aOR}=29.56 ; 95 \% \mathrm{CI}: 3.47-251.52 ; \mathrm{p}=0.002)$ with the presence of DRM in respondents. ARV substitution history was not associated with the presence of DRM. There were 17 respondents $(6.3 \%)$ carrying HIV-1 DRM, with M184V/I (11 sequences) as the most frequent pattern of NRTI resistance, and K103 (9 sequences) as that of NNRTI resistance.

\section{CONCLUSION}

This study demonstrated that viral load at the time of the study was the most influential determinant factor for the presence of DRM in PLWHA.

Keywords: Antiretroviral, drug resistance mutation, Papua Barat Province
${ }^{1}$ Center of Health Research and Development Papua, Indonesia ${ }^{2}$ VIB Center for Medical Biotechnology, Department of Biomolecular Medicine, Ghent University, Ghent, Belgium

\section{Correspondence:}

Hotma Martogi Lorensi Hutapea Jl. A. Yani No.48, Jayapura Phone (0967) 534389 Fax: (0967) 534697 Email: hotmahutapea@litbang.kemkes.go.id ORCID ID: 0000-0002-7099-3891

This open access article is distributed under a Creative Commons AttributionNon Commercial-Share Alike 4.0 International License

Date of first submission, June 14, 2021 Date of final revised submission, November 22, 2021

Date of acceptance, November 30 , 2021

Cite this article as: Hutapea HML, Kridaningsih TN, Prasetyo KH, Antwi MB. Viral load as a risk factor of reverse transcriptase inhibitor drug resistance mutation in antiretroviraltreated people living with HIV/AIDS. Univ Med 2021;40:243-51. doi: 10.18051/UnivMed.2021.v40.243253. 


\section{INTRODUCTION}

Human immunodeficiency virus type 1 (HIV-1) infection remains one of many major health challenges in Indonesia, including Papua Barat Province. ${ }^{(1,2)}$ Human immunodeficiency virus type 1 is a retrovirus with lack of proofreading activity of reverse transcriptase (RT). As a result, HIV-1 has high genetic diversity due to its high mutation rate. The manifestation of this characteristic is the presence of mutation which is caused by specific selection by antiretrovirals. ${ }^{(3-6)}$ Another impact of this dynamic diversity is the presence of natural polymorphisms in HIV-1. These polymorphisms by themselves are not significantly affecting viral susceptibility changes to antiretrovirals (ARV); however, in combination with drug resistance mutations (DRM) they might increase or decrease the effect of ARV on the virus. ${ }^{(7)}$

Antiretroviral therapy has been used to decrease the infection and increase the health quality of people living with HIV/AIDS (PLWHA). The major target of ARV therapy is HIV-1 RT. There are two classes of ARV, namely nucleotide reverse transcriptase inhibitors (NRTI), and non-nucleotide reverse transcriptase inhibitors (NNRTI). An effective life-long drug adherence is required to achieve successful treatment. ${ }^{(4,8)}$ Higher risk in developing DRM has been associated with low drug adherence. ${ }^{(9-12)}$

Drug resistance mutation is one of the major problems in ARV therapy management, especially in upscaling the ARV coverage program. In 2017 in Papua Province, there were 5.16\% DRM cases reported with a dominant mutation in HIV-1 RT amino acid 184 which changed from methionine to valine or isoleucine (M184V/I) in NRTI DRM patients. ${ }^{(13)} \mathrm{M} 184 \mathrm{~V} / \mathrm{I}$ reduces the susceptibility of HIV-1 to lamivudine (3TC) and emtricitabine (FTC), but increases its susceptibility to zidovudine (AZT) and tenofovir (TDF). Another major mutation called $\mathrm{K} 103 \mathrm{~N}$ is prevalent in NNRTI DRM patients. This mutation decreases the susceptibility of HIV-1 to nevirapine (NPV) and efavirenz (EFV). Combinations of M184V/I and $\mathrm{K} 103 \mathrm{~N}$ in individual patients are commonly identified. Several important mutations have been studied that affect viral susceptibility to ARV. ${ }^{(14}$ 16)

Monitoring of the $\mathrm{CD} 4$ cell count is important to predict the severity of HIV-1 infection during ARV therapy. Low CD4 cell counts are frequently associated with the presence of opportunistic infections, advanced disease, and death. ${ }^{(17)}$ During ARV therapy, the viral load decreases, which improves the immune response, as shown by the increased CD4 cell count. ${ }^{(9,17)}$ The success of ARV therapy is measured by the clinical appearance of the patients, the reduction in viral load to an undetectable level, and CD4 cell counts above 200 cells $/ \mathrm{mL}$.

Papua Barat province originally consisted of the 3 districts Manokwari, Sorong, and Fakfak, and by 2010 was administratively divided into 10 districts. ${ }^{(18)}$ The total population of Papua Barat has grown by approximately $32.94 \%$ from 2010 to 2020. The HIV cases in Papua Barat Province in 2019 cumulatively comprised 697 cases with AIDS case rate of 176.32. Based on the AIDS case rate, Papua Barat has the third highest rate in Indonesia, while the national AIDS case rate is 38.93. ${ }^{(19)}$ The majority of HIV-1 cases in Papua Barat are found in Manokwari, Sorong, and Fakfak. Particularly, PLWHA in Manokwari, Sorong, and Fakfak are treated with a fixed dose combination (FDC) containing tenofovir (TDF), emtricitabine (FTC), and efavirenz (EFV) in a single tablet. The Ministry of Health reported that CRF01_AE is the most predominant HIV-1 variant in most regions in Indonesia and that subtype B is common in Papua and Papua Barat. ${ }^{(20)}$

A study showed that detection of resistance and of certain DRM during virological failure of ART has a small but significant favorable effect on CD4 decline. ${ }^{(17)}$ By contrast, an European study involving 6180 individuals with a resistance test prior to starting antiretroviral treatment accessing care in HIV clinics showed that there was no definitive evidence that DRM influenced CD4+ cell decline. ${ }^{(21)}$ Viral loads and CD4 counts 
were found to be associated with HIV-1 drug resistance. (22) It is important to understand the interplay of factors associated with HIV drug resistance, especially in low to middle-income countries (LMICs) where there is limited access to viral load testing. ${ }^{(23)}$

Therefore, to understand the relationship of CD4 cell count and viral load with the presence of DRM, the aim of this study was to determine the association of clinical and ARV therapy related variables (CD4 cell count collected at the time of the study, changes in CD4 cell count, viral load, drug adherence, and regimen substitution history) with the presence of DRM in ARV-treated PLWHA in Papua Barat Province. We also identified dominant DRMs and important polymorphisms in these DRM sequences to determine the potential resistance to the FDC according to the HIV-1 ARV resistance database.

\section{METHODS}

\section{Research design}

This was a cross-sectional study on PLWHA in the care, service, and therapy (CST) clinics of government hospitals in Fakfak, Manokwari, and Sorong City, Papua Barat Province. This study was conducted over a 10month period from February to December 2019 in Fakfak, Manokwari, and Sorong, Papua Barat Province.

\section{Study subjects}

The population in this study consisted of PLWHA registered in the CST clinics, who were older than 18 years, and had received therapy for more than 6 months. An important inclusion criterion was that the respondents should be healthy at the time of data collection.

\section{Sample size determination}

This study noted from previous research that HIV-1 subtyping is successfully identified in $98 \%$ of patients by sequencing. ${ }^{(24)}$ The sample size determination for each study area was performed with the Lemeshow formula:

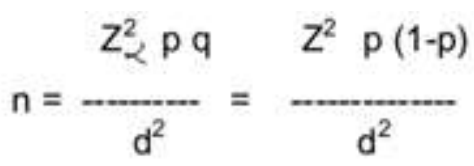

$\mathrm{n} \quad=$ sample size

$\mathrm{Z} 2 \alpha=95 \%$ level of confidence: 1.96

$\mathrm{p}=$ population proportion estimation $(0.98) ; \mathrm{q}=0.02$

$\mathrm{d}=$ permissible error $(0.01)$

Based on the calculations, at least 84 subjects were required in each studied area. The total number of subjects obtained was 269 PLWHA selected by consecutive sampling.

\section{Laboratory analysis}

Data and specimen collection was conducted in 2 rounds (of 14 days each) in the clinic. Therapy initiation CD4 cell count was copied from the patient's medical record by authorized health workers in the clinic. Blood specimen collection was performed by competent and qualified health workers assigned by the head of the CST clinic. The blood specimens were divided into 3 aliquots to obtain whole blood, serum, and plasma. The whole blood was used to measure CD4 cell count, performed on the same day using PIMA ${ }^{\mathrm{TM}}$-Alere (Germany). Serum and plasma were stored in a temperaturecontrolled freezer before shipment to Jayapura for viral load measurement and mutation identification. Sample shipment was conducted according to the 3-layer packaging and cold chain standard.

The serum was used to measure viral load by using a viral load analyzer with the AccuPower ${ }^{\circledR}$ HIV-1 Quantitative RT-PCR Kit (Bioneer, Republic of Korea). Mutation identification was performed on selected samples with a viral load of $>1000$ copies $/ \mathrm{mL}$. The reverse transcription-polymerase chain reaction (RT-PCR) was nested by using WHO HIV-DR primers ${ }^{(25)}$ that partially amplified the RT genes using the high fidelity enzyme mix SuperScript III One-Step RT-PCR System with Platinum 
Taq ${ }^{\circledR}$ (Invitrogen). PCR for sequencing was performed using the inner primer pair, and reading of the product was performed using the Sanger method. Each sample produced 2 sequences which were complementary to each other. BioEdit sequence alignment editor was used for trimming, editing, and creating the consensus sequences. The consensus sequence for each sample was submitted to the website http:// hiv.db.stanford.edu for DRM analysis. The output from the website was used as the reference of mutation identified in the patients and its clinical implication regarding ARV therapy.

\section{Statistical analysis}

Patients' data, CD4 cell count, viral load measurement and mutation status were added to a spreadsheet and descriptively analyzed using SPSS version 21. Respondents' medical data, CD4 counts, viral loads, and DRM were analyzed using simple and multiple logistic regression. A statistical result was considered significant when $\mathrm{p}<0.05$.

\section{Ethical clearance}

This study was ethically approved by the Health Research Ethics Committee, National Institute of Health Research and Development (HREC - NIHRD) under number LB.02.01/2/ KE.008/2019.

\section{RESULTS}

Demographic characteristics of respondents

The majority of the respondents was female (54.3\%), Papuan (53.0\%), and older than 35 years $(80.5 \%)$. Most of the respondents were married $(78.6 \%)$, graduated from higher education (74.0\%), and employed (66.9\%) (Table 1). The CD4 cell count measured in this study showed that most of the respondents $(81.4 \%)$ had CD4 T-cell counts of more than 200 cell $\mathrm{s} / \mathrm{mL}$ and viral loads below 1000 copies/mL was $210(78.1 \%)$ ((Table 1).
Table 1. Characterization of the study population $(\mathrm{n}=269)$

\begin{tabular}{|c|c|}
\hline Variables & n $(\%)$ \\
\hline \multicolumn{2}{|l|}{ Sex } \\
\hline Female & $146(54.3)$ \\
\hline Male & $123(45.7)$ \\
\hline \multicolumn{2}{|l|}{ Ethnicity } \\
\hline Papuan & $123(45.7)$ \\
\hline Non-Papuan & $146(54.3)$ \\
\hline \multicolumn{2}{|l|}{ Age (years) } \\
\hline$\leq 35$ & $143(53.2)$ \\
\hline$>35$ & $126(46.8)$ \\
\hline \multicolumn{2}{|l|}{ Marital status } \\
\hline Married & $209(77.7)$ \\
\hline Single & $60(22.3)$ \\
\hline \multicolumn{2}{|l|}{ Formal education } \\
\hline Elementary to junior high & $69(26.0)$ \\
\hline Senior high to higher education & $196(74.0)$ \\
\hline \multicolumn{2}{|l|}{ Occupation } \\
\hline Employed & $88(33.1)$ \\
\hline Unemployed & $178(66.9)$ \\
\hline \multicolumn{2}{|l|}{ CD4 count (cells/mL) } \\
\hline$\leq 200$ & $50(18.6)$ \\
\hline$>200$ & $219(81.4)$ \\
\hline \multicolumn{2}{|l|}{ CD4 count before therapy (cells $/ \mathrm{mL}$ ) } \\
\hline$\leq 200$ & $74(27.5)$ \\
\hline$>200$ & $80(29.7)$ \\
\hline Not available & $115(42.8)$ \\
\hline \multicolumn{2}{|l|}{$\begin{array}{l}\text { CD4 count changes after therapy } \\
\text { (cells } / \mathrm{mL} \text { ) }\end{array}$} \\
\hline $\begin{array}{l}\text { Decreased to } \leq 200 \text { or } \\
\text { maintained } \leq 200\end{array}$ & $22(14.2)$ \\
\hline $\begin{array}{l}\text { Increased to }>200 \text { or } \\
\text { maintained }>200\end{array}$ & $132(85.8)$ \\
\hline \multicolumn{2}{|l|}{ Viral load (copies/mL) } \\
\hline$\leq 1,000$ & $210(78.1)$ \\
\hline$>1,000$ & $59(21.9)$ \\
\hline \multicolumn{2}{|l|}{$\begin{array}{l}\text { Mutation associated to drug } \\
\text { resistance }\end{array}$} \\
\hline Yes & $17(6.3)$ \\
\hline No & $252(93.7)$ \\
\hline ART-naïve & $0(0.0)$ \\
\hline ART failure & $43(16.0)$ \\
\hline \multicolumn{2}{|l|}{ Therapy adherence } \\
\hline Moderate - poor & $39(14.5)$ \\
\hline Good & $230(85.5)$ \\
\hline \multicolumn{2}{|l|}{ Antiretroviral therapy } \\
\hline FDC & $221(82.2)$ \\
\hline Non-FDC & $46(17.1)$ \\
\hline NA & $2(0.7)$ \\
\hline \multicolumn{2}{|l|}{ Length of therapy } \\
\hline$\leq 12$ months & $197(73.2)$ \\
\hline$>12$ months & $45(16.7)$ \\
\hline NA & $27(10.1)$ \\
\hline \multicolumn{2}{|l|}{ Regimen substitution } \\
\hline Yes & 32 (11.9) \\
\hline No & $237(88.1)$ \\
\hline
\end{tabular}


Table 2. Profile of patients with DRM

\begin{tabular}{|c|c|c|c|c|c|c|c|c|}
\hline \multirow{3}{*}{ Variables } & \multicolumn{6}{|c|}{ Drug resistant } & \multirow{2}{*}{\multicolumn{2}{|c|}{$\begin{array}{c}\text { Drug sensitive to } \\
\text { both NNRTI and } \\
\text { NRTI }\end{array}$}} \\
\hline & \multicolumn{2}{|c|}{ NNRTI } & \multicolumn{2}{|c|}{ NRTI } & \multicolumn{2}{|c|}{$\begin{array}{c}\text { NNRTI or } \\
\text { NRTI }\end{array}$} & & \\
\hline & $\mathbf{n}$ & $\%$ & $\mathbf{n}$ & $\%$ & $\mathbf{n}$ & $\%$ & $\mathbf{n}$ & $\%$ \\
\hline Papuan & 11 & 4.1 & 6 & 2.2 & 6 & 2.2 & 117 & 43.5 \\
\hline Non-Papuan & 5 & 1.9 & 5 & 1.9 & 4 & 1.5 & 135 & 50.2 \\
\hline \multicolumn{9}{|l|}{ Gender } \\
\hline Male & 6 & 2.2 & 4 & 1.5 & 3 & 1.1 & 117 & 43.5 \\
\hline Female & 10 & 3.7 & 7 & 2.6 & 7 & 2.6 & 135 & 50.2 \\
\hline \multicolumn{9}{|l|}{ Adherence } \\
\hline Yes & 13 & 4.8 & 9 & 3.3 & 9 & 3.3 & 216 & 80.3 \\
\hline No & 3 & 1.1 & 2 & 0.7 & 1 & 0.4 & 36 & 13.4 \\
\hline $\mathrm{CD} 4<200$ cells $/ \mathrm{mL}$ & 11 & 4.1 & 8 & 3.0 & 8 & 3.0 & 63 & 23.4 \\
\hline $\begin{array}{l}\mathrm{CD} 4<200 \text { cells } / \mathrm{mL} \\
\text { before treatment and } \\
\text { maintained at }<200 \\
\text { cells } / \mathrm{mL} \text { after treatment }\end{array}$ & 5 & 3.2 & 4 & 2.6 & 4 & 2.6 & 14 & 9.1 \\
\hline $\begin{array}{l}\text { Viral load }>1000 \\
\text { copies } / \mathrm{mL}\end{array}$ & 14 & 5.2 & 9 & 3.3 & 8 & 3.0 & 43 & 16.0 \\
\hline
\end{tabular}

Note : DRM = drug resistance mutations; NNRTI : non-nucleotide reverse transcriptase inhibitors; NRTI : nucleotide reverse transcriptase inhibitors

According to the ARV therapy adherence history, the majority $(85.5 \%)$ of the respondents in this study was categorized as having good adherence. Most of the respondents $(82.2 \%)$ was receiving FDC for treatment and $73.2 \%$ had undergone therapy for more than 12 months. The majority of respondents $(88.1 \%)$ never had regimen substitution for their therapy (Table 1). Based on their DRM status, the majority of respondents of NNRTI classes was Papuan (4.1\%), female $(3.7 \%)$, and $4.1 \%$ had a CD4 cell count below 200 cells $/ \mathrm{mL}$ (Table 2 ).

\section{Association of clinical and therapy characteristics of respondents with DRM presence in RT nucleotide sequence}

Simple logistic regression analysis on variables regarding respondents' clinical data showed that there was a significant association between CD4 cell count and DRM ( $p<0.001)$. Respondents with CD4 cell counts of less than 200 cells $/ \mathrm{mL}$ had a higher probability of carrying HIV-1 with DRM compared to respondents with CD4 cell counts of more than 200 cells $/ \mathrm{mL}$ $(\mathrm{OR}=.38 .45 ; 95 \%$ CI:5.19-285.24; $\mathrm{p}=<0.001)$.
In this study, a significant association was also found between viral load and DRM $(\mathrm{p}<0.001)$. This study showed that respondents with viral loads of more than 1000 copies $/ \mathrm{mL}$ had higher chances of developing DRM $(\mathrm{OR}=56.95 ; 95 \%$ CI: 7.71-420.61); $\mathrm{p}<0.001)$ than did respondents with viral loads below 1000 copies/mL. However, there was no significant association between therapy adherence and DRM, and between regimen substitution and DRM. The multiple logistic regression analysis showed the viral load to be the most influential determinant of DRM $(\mathrm{aOR}=29.56$;95\% CI: 3.47 251.52; $\mathrm{p}=0.002)$ (Table 3$)$.

\section{Identification of Subtype, DRM pattern, and polymorphisms}

Among 59 respondents with a viral load of more than 1000 copies/mL, 30 RT partial gene sequences $(50.8 \%)$ were successfully amplified. However, only 21 sequences $(70.0 \%)$ were of sufficiently good quality to be analyzed further. Subtype B was dominant in this study (10 out of 21 sequences, $47.6 \%$ ), followed by Circulating Recombinant Form (CRF) 01_AE (7 out of 21 
Table 3. Factors associated with RTI- DRM among participants (using simple and multiple logistic regression)

\begin{tabular}{|c|c|c|c|c|c|}
\hline Factors & $\begin{array}{c}\text { DRM rate } \\
(\mathbf{n}, \%)\end{array}$ & $\begin{array}{c}\text { Crude OR } \\
(95 \% \text { CI }) \\
\end{array}$ & $p$ value & $\begin{array}{c}\text { Adjusted OR } \\
(95 \% \mathrm{CI}) \\
\end{array}$ & p value \\
\hline \multicolumn{6}{|l|}{ CD4 count $($ cells $/ \mu \mathrm{L})$} \\
\hline$<200$ & $16(5.94)$ & 38.48 & $<0.001$ & 12.47 & 0.023 \\
\hline$\geq 200$ & $1(0,003)$ & $(5.19-285.24)$ & & $(1.45-107.39)$ & \\
\hline \multicolumn{6}{|l|}{ Viral load (copies/mL) } \\
\hline$\leq 1,000$ & $1(0.003)$ & 56.95 & $<0.001$ & 29.56 & 0.002 \\
\hline$>1,000$ & $16(5.94)$ & $(7.71-420.61)$ & & $(3.47-251.52)$ & \\
\hline \multicolumn{6}{|l|}{ Therapy adherence } \\
\hline Moderate - poor & $3(1.1)$ & 1.26 & & 0.56 & \\
\hline Good & $14(5.2)$ & $(0.38-4.19)$ & 0.70 & $(0.13-2.48)$ & 0.52 \\
\hline \multicolumn{6}{|l|}{ Regimen substitution } \\
\hline Yes & $1(0.003)$ & 0.46 & & 0.28 & \\
\hline No & $16(5.94)$ & $(0.06-3.37)$ & 0.45 & $(0.03-2.56)$ & 0.26 \\
\hline
\end{tabular}

Note : DRM : drug resistance mutations; $\mathrm{OR}=$ odds ratio

sequences, 30\%) (Table 4). The DRM patterns were identified in 17 sequences. M184V/I was the most frequent pattern identified for NRTI resistance (11 respondents, 64.7\%). Mutation $\mathrm{K} 103 \mathrm{~N}$ was the most frequent pattern ( 9 respondents, 52.9\%) identified for NNRTI resistance (Figure 1). Of the important natural polymorphisms, K122E was identified in almost all sequences (14 respondents, $70.0 \%$ ), and G335D in one sequence (Table 4).

\section{DISCUSSION}

Based on viral load and CD4 cell count data, the majority of respondents in this study were successfully treated by ARV therapy. The significant association between CD4 cell count and the presence of DRM was in accordance with previous studies..$^{(9,17,26)}$ Our study showed that respondents with CD4 cell counts below 200 cells $/ \mathrm{mL}$ had a 38.45 times higher probability of carrying HIV-1 with DRM than did those with CD4 cell counts above 200 cells $/ \mathrm{mL}$. Other studies have shown that CD4 cell count may be a strong predictor of DRM present in PLWHA undergoing ARV therapy. ${ }^{(13)}$ Monitoring the CD4 cell count regularly may help in predicting the presence of DRM in PLWHA in Papua Barat, since detecting DRM is intricate and challenging. Another benefit of measuring CD4 cell counts regularly is to detect reductions in CD4 cell count and to anticipate the progression of infection into acquired immunodeficiency syndrome (AIDS) diseases. ${ }^{(27)}$

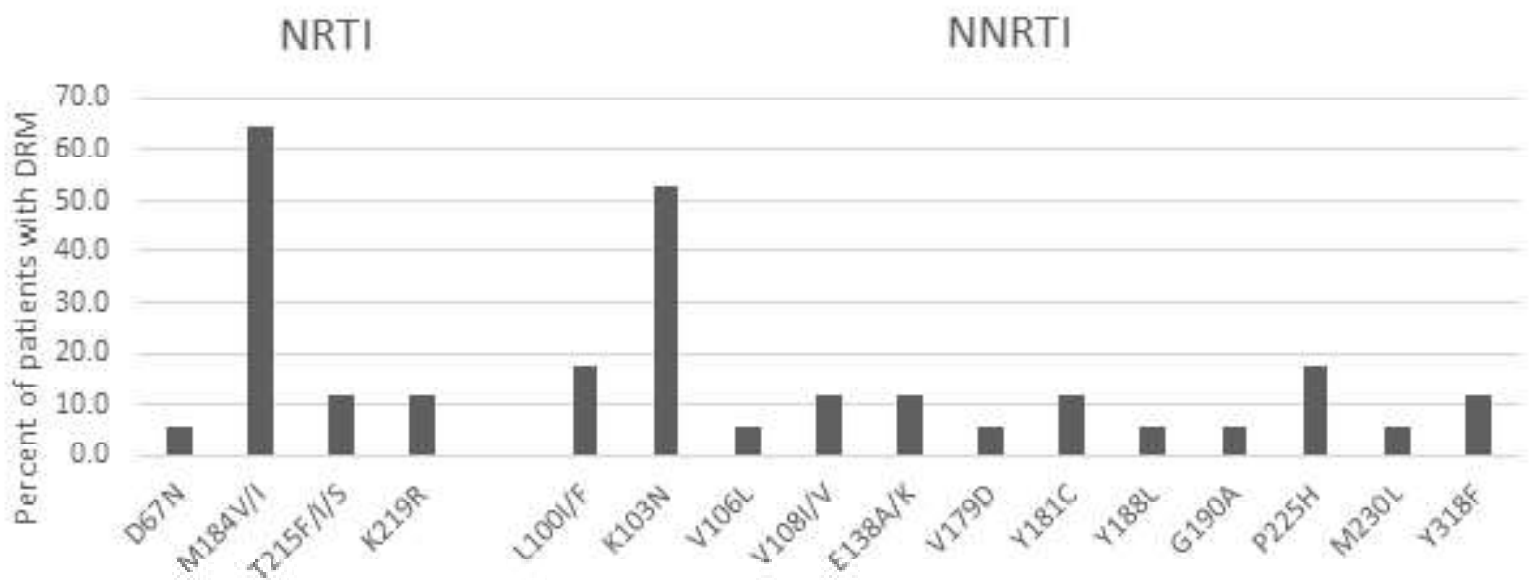

Figure 1. The distribution of DRM pattern in Papua Barat 
Table 4. Subtype, polymorphisms, and DRM identified from patients and clinical implication

\begin{tabular}{|c|c|c|c|c|c|c|}
\hline $\begin{array}{c}\text { Sample } \\
\text { Code }\end{array}$ & Subtype & $\begin{array}{c}\text { NRTI } \\
\text { mutation }\end{array}$ & $\begin{array}{c}\text { NRTI } \\
\text { resistance }\end{array}$ & $\begin{array}{c}\text { NNRTI } \\
\text { mutation }\end{array}$ & $\begin{array}{l}\text { NNRTI } \\
\text { resistance }\end{array}$ & Polymorphism \\
\hline 910101 & $\mathrm{~B}$ & - & - & E138EA & ETR, RPV & $\begin{array}{l}\text { K122E, I135V, I142IM, S162C, I195T, } \\
\text { T200A, L228M, V245E, I293V, P313T }\end{array}$ \\
\hline 910114 & CRF01_AE & $\begin{array}{l}\text { M1841, } \\
\text { T215TFIS, } \\
\text { K219Q }\end{array}$ & $\begin{array}{l}\mathrm{ABC}, \mathrm{AZT} \\
\mathrm{FTC}, 3 \mathrm{TC}\end{array}$ & $\begin{array}{l}\text { V1081, } \\
\text { Y181C }\end{array}$ & $\begin{array}{l}\text { DOR, EFV } \\
\text { ETR, NVP, } \\
\text { RPV }\end{array}$ & $\begin{array}{l}\text { K122E, I135V, S162C, I195T, L228M, } \\
\text { V245E, E297EA, P313T }\end{array}$ \\
\hline 910143 & A & - & - & - & - & $\begin{array}{l}\text { K122E, D123G, I135T, R172K, K173KR, } \\
\text { Q174K, D177E, I178M, L193F, E194P, } \\
\text { Q207D, V245E, S251N, A272P, T286A, } \\
\text { E291K }\end{array}$ \\
\hline 910166 & B & - & - & E138EK & $\begin{array}{l}\text { DOR, EFV } \\
\text { ETR, NVP, } \\
\text { RPV }\end{array}$ & $\begin{array}{l}\text { D121Y, K122E, I135V, I142IMT, S162C, } \\
\text { G196E, Q207QHR, R211G, L228M, } \\
\text { I293V }\end{array}$ \\
\hline 910167 & B & - & - & - & - & $\begin{array}{l}\text { K122E, I135V, S162C, I195T, L228M, } \\
\text { V245E, E297EA, P313T }\end{array}$ \\
\hline 910179 & A & - & - & $\begin{array}{l}\text { L1001, } \\
\text { K103N }\end{array}$ & $\begin{array}{l}\text { DOR, EFV } \\
\text { ETR, NVP, } \\
\text { RPV }\end{array}$ & $\begin{array}{l}\text { K122E, D123S, R172K, K173I, Q174K, } \\
\text { D177DEG, E194P, T200I, Q207A, R211N, } \\
\text { V245E, A272G, K281R, T286A, E291D, } \\
\text { V292I, I293V, L295X, T296K, E297K, } \\
\text { E305V, N306S, I309T, L310F, E312S }\end{array}$ \\
\hline 910508 & B & - & - & - & - & $\begin{array}{l}\text { T107S, K122E, S162C, T200I, R211K, } \\
\text { V292I, I293V, L301I, Y319F }\end{array}$ \\
\hline 910516 & B & M184V & $\begin{array}{l}\mathrm{ABC}, \mathrm{AZT} \\
\mathrm{FTC}, 3 \mathrm{TC}\end{array}$ & - & - & $\begin{array}{l}\text { K103R, I135V, S162C, I178M, Q207E, } \\
\text { R211K, L228M, I293V }\end{array}$ \\
\hline 910520 & B & M184V & $\begin{array}{l}\mathrm{ABC}, \mathrm{AZT} \\
\mathrm{FTC}, 3 \mathrm{TC}\end{array}$ & $\begin{array}{l}\mathrm{K} 103 \mathrm{~N}, \\
\mathrm{P} 225 \mathrm{H}\end{array}$ & $\begin{array}{l}\text { DOR, EFV } \\
\text { ETR, NVP }\end{array}$ & $\begin{array}{l}\text { K102Q, D123E, I135L, S162C, T200I, } \\
\text { R211K, L228Q, A272P, V276I, L283I, } \\
\text { V292I, I293V }\end{array}$ \\
\hline 910528 & B & - & - & $\begin{array}{l}\text { L100F, } \\
\text { K103N }\end{array}$ & $\begin{array}{l}\text { DOR, EFV } \\
\text { ETR, NVP }\end{array}$ & $\begin{array}{l}\text { K122E, D123N, T128TS, S162C, I178L, } \\
\text { T200I, H208R, R211K, L228F, V245E, } \\
\text { E248D, A272P, V292I, I293V, V317A }\end{array}$ \\
\hline 910530 & B & - & - & $\begin{array}{l}\text { K103N, } \\
\text { V108VI }\end{array}$ & $\begin{array}{l}\text { DOR, EFV } \\
\text { ETR, NVP }\end{array}$ & K102Q, D123E \\
\hline 910532 & CRF01_AE & M184V & $\begin{array}{l}\mathrm{ABC}, \mathrm{AZT} \\
\mathrm{FTC}, 3 \mathrm{TC}\end{array}$ & $\begin{array}{l}\mathrm{P} 225 \mathrm{H}, \\
\mathrm{K} 103 \mathrm{~N}\end{array}$ & $\begin{array}{l}\text { DOR, EFV } \\
\text { ETR, NVP }\end{array}$ & $\begin{array}{l}\text { K122E, D123N, K173V, Q174K, V179I, } \\
\text { I202V, Q207A, R211S, K238R, V245E, } \\
\text { A272P, E291D, V292I, I293V, E312N, } \\
\text { I329V. }\end{array}$ \\
\hline 910701 & CRF01_AE & M1841 & $\begin{array}{l}\mathrm{ABC}, \mathrm{AZT} \\
\mathrm{FTC}, 3 \mathrm{TC}\end{array}$ & $\begin{array}{l}\text { Y318F, } \\
\text { L1001, } \\
\text { K103N }\end{array}$ & $\begin{array}{l}\text { DOR, EFV } \\
\text { ETR, NVP }\end{array}$ & $\begin{array}{l}\text { K122E, D123S, T139K, K173I, Q174K, } \\
\text { D177E, T200E, Q207A, R211K, K238R, } \\
\text { V245E, T286A, I293V, E312A, I326V, } \\
\text { I329V, K331N }\end{array}$ \\
\hline 910764 & A & - & - & - & - & $\begin{array}{l}\text { K173I, Q174K, Q197QE, Q207A, R211S, } \\
\text { V245E, T286A, I293V, E312T, S322A, } \\
\text { L325V, I329Y, Q330R, G335D. }\end{array}$ \\
\hline 910749 & B & - & - & K103N & $\begin{array}{l}\text { DOR, EFV } \\
\text { ETR, NVP }\end{array}$ & G112A, K122E, D123S \\
\hline 910765 & A & $\begin{array}{l}\text { M184V, } \\
\text { T215F, } \\
\text { M41L, } \\
\text { D67N, }\end{array}$ & $\begin{array}{l}\mathrm{ABC}, \mathrm{AZT} \\
\mathrm{FTC}, 3 \mathrm{TC}\end{array}$ & $\begin{array}{l}\text { V179D, } \\
\text { M230L, } \\
\text { V106L }\end{array}$ & $\begin{array}{l}\text { DOR, EFV } \\
\text { ETR, NVP, } \\
\text { RVP }\end{array}$ & $\begin{array}{l}\text { V106L, K122E, D123N, I135K, K173T, } \\
\text { Q174K, G196E, I202V, Q207A, R211K, } \\
\text { L228H, V245E, L283I, E291D, V292I, } \\
\text { I293V, K311R, E312A, I329T }\end{array}$ \\
\hline 910781 & CRF01_AE & M184I & $\begin{array}{l}\mathrm{ABC}, \mathrm{AZT} \\
\mathrm{FTC}, 3 \mathrm{TC}\end{array}$ & $\begin{array}{l}\text { K103N, } \\
\text { Y318F }\end{array}$ & $\begin{array}{l}\text { DOR, EFV } \\
\text { ETR, NVP }\end{array}$ & $\begin{array}{l}\text { K122E, D123S, T139K, K173I, Q174K, } \\
\text { D177E, T200E, Q207A, R211K, K238R, } \\
\text { V245E, T286A, I293V, E312A, I326V }\end{array}$ \\
\hline 9105105 & CRF01_AE & M184V & $\begin{array}{l}\text { ABC, AZT, } \\
\text { FTC, 3TC }\end{array}$ & $\begin{array}{l}\text { Y181C, } \\
\text { G190A }\end{array}$ & $\begin{array}{l}\text { DOR, EFV } \\
\text { ETR, NVP, } \\
\text { RVP }\end{array}$ & $\begin{array}{l}\text { I142M, S163C, K173I, Q174K, Q207A, } \\
\text { R211S, V245E, A272P, V276I, T286A, } \\
\text { E291D, I293V, E312N, I326V, I329R }\end{array}$ \\
\hline 910149 & CRF01_AE & $\begin{array}{l}\text { M184V, } \\
\text { K219KR }\end{array}$ & $\begin{array}{l}\text { ABC, AZT, } \\
\text { FTC, 3TC }\end{array}$ & $\mathrm{P} 225 \mathrm{H}$ & $\begin{array}{l}\text { DOR, EFV } \\
\text { ETR, NVP }\end{array}$ & $\begin{array}{l}\text { D123E, I135M, S162C, T200TA, Q207E, } \\
\text { L228KM, I257L, A272P, I293V, K311N, } \\
\text { E312Q, H315Q, V317A }\end{array}$ \\
\hline 910150 & CRF01_AE & M184 I & $\begin{array}{l}\mathrm{ABC}, \mathrm{AZT} \\
\mathrm{FTC}, 3 \mathrm{TC}\end{array}$ & Y188L & $\begin{array}{l}\text { DOR, EFV } \\
\text { ETR, NVP, } \\
\text { RVP }\end{array}$ & $\begin{array}{l}\text { K122E, D123N, K173I, Q174K, D177E, } \\
\text { V189I, E194P, T200I, E203D, Q207A, R2 } \\
\text { 11S, K238R, V245E, A272G, K281R, T28 } \\
\text { 6A, E291D, V292I, I293V, E297EK, E312 } \\
\text { N, I326V, I329V }\end{array}$ \\
\hline 910714 & B & M184V & $\begin{array}{l}\mathrm{ABC}, \mathrm{AZT} \\
\mathrm{FTC}, 3 \mathrm{TC}\end{array}$ & $\begin{array}{l}\mathrm{K} 103 \mathrm{~N} \\
\mathrm{P} 225 \mathrm{H}\end{array}$ & $\begin{array}{l}\text { DOR, EFV } \\
\text { ETR, NVP }\end{array}$ & $\begin{array}{l}\text { I142M, S163C, K173I, Q174K, Q207A, } \\
\text { R211S, V245E, A272P, V276I, T286A, } \\
\text { E291D, I293V, E312N, I326V, I329R }\end{array}$ \\
\hline
\end{tabular}


The CD4 cell count of patients with good virological control will increase by approximately $50-150$ cells $/ \mathrm{mm}^{3}$ per year. ${ }^{(26)}$ A study conducted by Schultze et al., ${ }^{(17)}$ showed that DRM at a particular site in RT might contribute to the stable CD4 count during therapy. In our study, there was a significant association between the difficulty of increasing the CD4 cell count to more than 200 cells $/ \mathrm{mL}$ and the presence of DRM. The group of respondents with difficulties of increasing their CD4 cell count had an approximately 48 times higher probability of carrying HIV-1 with DRM compared to the group who had their CD4 cell count increased to or maintained above 200 cells $/ \mathrm{mL}$. The mutations M184V, D67N and $\mathrm{T} 215 \mathrm{Y}$ were associated with reduced CD4 count, whereas mutations V179D and L74V were associated with a steeper decline in CD4 count. In our study, M184V was the most frequent mutation in the NRTI class of ARV, and one respondent carried HIV-1 with mutation V179D (Table 4).

Another strong predictor of HIV-1 infection progression is viral load. The gold standard is to measure the number of RNA copies in the patient's sample. ${ }^{(28,29)} \mathrm{A}$ patient with a viral load above 1000 copies $/ \mathrm{mL}$ in two consecutive tests within 3 months after at least 6 months of therapy and with a CD4 cell count below 200 cells $/ \mathrm{mL}$ is considered to be experiencing treatment failure. ${ }^{(30)}$ In this study, the viral load was measured only once during the study, due to the lack of resources and certain equipment in Papua Barat Province. However, there was a significant association between viral load and the presence of DRM. Respondents with viral loads above 1,000 copies $/ \mathrm{mL}$ had a 56 times higher probability of carrying HIV-1 with DRM than those with less than 1,000 copies $/ \mathrm{mL}$, regardless of the number or pattern of the mutations. The immediate objective of ARV therapy is to inhibit viral replication and reduce the number of virus particles to undetectable levels. In this condition, ARV therapy is adequate and DRM will not develop. This can only be achieved by the patients' excellent adherence to ARV therapy.(7)

Patients' adherence to therapy is important to optimize the benefits of ARV therapy. Studies have revealed that good adherence was associated with an increase in CD4 T cells and a reduction in viral load. ${ }^{(12,31)}$ In our study, adherence was measured by the numbers of pills left after each month of therapy as counted by the health workers in the clinic. In our study, the moderate to poor adherence to therapy was not significantly associated with the presence of DRM. This result was inconsistent with a previous study which showed that adherence to ARV therapy was one of the important factors that affected the outcome of the therapy. The non-adherence behavior in taking therapy might cause viral rebound and was significantly associated with the presence of DRM. Nonadherence to taking ARV might also be a predictor of the severity of AIDS diseases. ${ }^{(32)}$ This inconsistent result might be due to difficulties in tracking the accurate numbers of pills taken by the patients daily that were faced by the physicians or assigned health workers.

The percentage of RTI-associated DRM was $6.3 \%$ and the most frequent pattern was M184V/I for NRTI-associated DRM and K103N for NNRTI. This was higher compared to the RTI-associated DRM percentage in Papua Province in 2017 (5.16\%). ${ }^{(13)}$ Several studies reported that mutations at M184 were the most frequently observed. ${ }^{(29-31)}$ Mutation $\mathrm{M} 184 \mathrm{~V} / \mathrm{I}$ confers high level resistance to lamivudine (3TC) and emtricitabine (FTC). Based on the guideline for ARV therapy for adults and adolescents, there are several regimen substitutions to be considered when M184V/I is detected in patients based on their clinical scenario. $^{(33)}$ Another major DRM identified in the present study was K103N which confers high level resistance to NNRTI classes of ARV and was the most frequent pattern identified in this study. This result is similar to the finding in Mexico. ${ }^{(34)}$ 
In our study, we also found natural polymorphisms associated with changes in the characteristic of the virus, for example K122E, I135V/I, and G335D. ${ }^{(14,34)} \mathrm{K} 122 \mathrm{E}$ was identified in almost all patients with or without any NRTI or NNRTI mutations. This was in line with another study reporting that $\mathrm{K} 122 \mathrm{E}$ was common in HIV1 infected patients with NRTI mutations. The accumulation of K122E with other polymorphisms or mutations might increase the numbers of NRTI and NNRTI mutations and contribute to higher levels of NRTI resistance. ${ }^{(4)}$ Another polymorphism identified in our study was G335D. A study conducted by Tanuma et al. ${ }^{(35)}$ showed that the presence of G335D polymorphism in HIV-1 CRF01_AE made the virus less susceptible to AZT in the background of M41L, L210W and T215Y (8.2 - to 23.2-fold) except for 3TC. The G335D polymorphisms increased viral susceptibility to AZT, ABC, and $\operatorname{TDF}(52.7,4.2$, and 2.4-fold respectively) in the background of D67N, K70R and T215F DRM. Our study highlighted that G335D was identified in patients without NRTI. How this polymorphism works without any mutation as the background is not clear. One limitation of this study was the inability to identify polymorphism G335 in all of the sequences due to the differences in length as a result of sequence 3'-end trimming.

Due to the limitation of continuity of CD4 cartridge supply, not all respondents were provided with a complete data set of CD4 cell counts. However, the DRM and polymorphism data obtained in this study is important in monitoring the usage of ARV in Papua Barat. This study might be useful in making recommendations that could contribute to reviewing treatment monitoring policy in Papua Barat.

\section{CONCLUSIONS}

This study highlighted the necessity of monitoring patients' CD4 cell count and viral load to reduce the possibility of DRM development. The presence of polymorphisms should be brought to attention with regard to the possibility that their combination with mutations might affect virus susceptibility to ARV.

\section{CONFLICT OF INTEREST}

Competing interests: No relevant disclosures.

\section{ACKNOWLEDGEMENT}

We gratefully acknowledge the cooperation of the health workers and patients involved in this study. We also thank the laboratory technicians who helped with the collection of laboratory data for this study.

\section{CONTRIBUTORS}

HMLH contributed to conception of the study, data acquisition, analysis and interpretation, drafting the manuscript, and revising the manuscript. TNK contributed to conception of the study, data acquisition, analysis and interpretation, and revising the manuscript. KHP contributed to data acquisition, analysis and interpretation. MBA contributed to revising the manuscript critically for important intellectual content. All authors have read and approved the final manuscript.

\section{REFERENCES}

1. Menteri Kesehatan RI. Rencana aksi nasional pengendalian HIV-AIDS tahun 2015-2019. Jakarta: Kementerian Kesehatan RI; 2015.

2. Pemerintah Provinsi Papua Barat Dinas Kesehatan. Profil kesehatan Papua Barat; 2018.

3. Abecasis AB, Wensing AMJ, Paraskevis D, et al. HIV-1 Subtype distribution and its demographic determinants in newly diagnosed patients in Europe suggest highly compartmentalized epidemics. Retrovirology 2013;10:1-13. DOI: 10.1186/1742-4690-10-7.

4. Melikian GL, Rhee SY, Varghese V, et al. Nonnucleoside reverse transcriptase inhibitor (NNRTI) cross-resistance: implications for preclinical evaluation of novel NNRTIs and 
clinical genotypic resistance testing. J Antimicrob Chemother 2014;69:12-20. DOI: 10.1093/jac/ dkt316.

5. Castro H, Pillay D, Cane P, et al. Persistence of HIV-1 transmitted drug resistance mutations. J Infect Dis 2013;208:1459-63. DOI: 10.1093/infdis/ jit345.

6. Quashie PK, Mesplede T, Han YS, et al. Characterization of the R263K mutation in HIV-1 integrase that confers low-level resistance to the second-generation integrase strand transfer inhibitor dolutegravir. J Virol 2012;86:2696-705. DOI: 10.1128/jvi.06591-11.

7. Krishnan KM, Amsavathani SK. Polymorphisms of HIV RT gene among the ART naive native drug exposed rural PLHA. J Glob Infect Dis 2012;4:110 3. DOI: $10.4103 / 0974-777 X .9677 .5$.

8. Palumbo PJ, Fogel JM, Hudelson SE, et al. HIV drug resistance in adults receiving early versus delayed antiretroviral therapy: HPTN 052. J Acquir Immune Defic Syndr 2018;77:484-91. DOI: 10.1097/QAI.0000000000001623.

9. Bock P, James A, Nikuze A, et al. Baseline CD4 count and adherence to antiretroviral therapy: a systematic review and meta-analysis. J Acquir Immune Defic Syndr 2016;73:514-21. DOI: 10.1097/QAI.0000000000001092.

10. Haberer JE, Sabin L, Amico KR, et al. Improving antiretroviral therapy adherence in resourcelimited settings at scale: a discussion of interventions and recommendations. J Int AIDS Soc 2017;20:21371-85. DOI: 10.7448/IAS.20.1. 21371.

11. Da W, Li X, Qiao S, et al. Evaluation of self-report adherence measures and their associations with detectable viral load among people living with HIV (PLHIV) in China. PLoS One 2018;13e0203032. DOI: 10.1371/journal.pone.0203032.

12. Ekong E, Ndembi N, Okonkwo P, et al. Epidemiologic and viral predictors of antiretroviral drug resistance among persons living with HIV in a large treatment program in Nigeria. AIDS Res Ther 2020;17:1-8. DOI: 10.1186/s12981-020-0261$\mathrm{z}$.

13. Hutapea HML, Fitriana E, Natalia EI, et al. Gambaran kasus mutasi terkait resistensi antiretroviral pada orang dengan HIV-AIDS (ODHA) di tiga Kabupaten / Kota di Provinsi Papua. Bul Penelit Kesehat 2018;46:199-206. DOI: 10.22435/bpk.v46i3.902.

14. Deletsu SD, Maina EK, Quaye O, Ampofo WK, Awandare GA, Bonney EY. High resistance to reverse transcriptase inhibitors among persons infected with human immunodeficiency virus type 1 subtype circulating recombinant form 02 AG in Ghana and on antiretroviral therapy. Medicine (Baltimore): 2020;99:e18777. DOI: 10.1097/ MD.0000000000018777.

15. Hong SY, Nachega JB, Kelley K, Bertagnolio S, Marconi VC, Jordan MR. The global status of HIV drug resistance: clinical and public-health approaches for detection, treatment and prevention. Infect Disord Drug Targets 2011;11:124-33.

16. Lodi S, Günthard HF, Dunn D, et al. Effect of immediate initiation of antiretroviral treatment on the risk of acquired HIV drug resistance. AIDS 2018;32:327-35.DOI: 10.1097/QAD.000000000000 1692.

17. Schultze A, Paredes R, Sabin C, et al. The association between detected drug resistance mutations and CD4+ T-cell decline in HIV-positive individuals maintained on a failing treatment regimen. Antivir Ther 2018;23:105-16. DOI: 10.3851/IMP3178.

18. Badan Pusat Statistik Provinsi Papua Barat. Hasil sensus penduduk 2020 Provinsi Papua Barat. Manokwari: Berita Resmi Statistik; 2020.

19. Kementerian Kesehatan RI. Direktorat Jenderal Pengendalian Penyakit Pusat Data dan Informasi. HIV AIDS . Kesehatan 2020. Jakarta: Pusat Data dan Informasi;2020.

20. Ministry of Health Republic of Indonesia. HIV epidemiology review, Indonesia 2016. Jakarta : Directorate General of Disease Prevention and Control; 2017.

21. Schultze A, Torti C, Cozzi-Lepri A, et al. for the European Transmitted Drug Resistance collaboration (EU-TDR). The effect of primary drug resistance on CD4R cell decline and the viral load set-point in HIV-positive individuals before the start of antiretroviral therapy. AIDS 2019; 33:315-26.DOI:10.1097/QAD.0000000000002046.

22. Lu X, Zhao H, Zhang Y, et al. HIV 1 drug resistant mutations and related risk factors among HIV 1 positive individuals experiencing treatment failure in Hebei Province, China. AIDS Res Ther 2017;14:4. DOI 10.1186/s12981-017-0133-3.

23. Rossouw TM, Feucht UD, Melikian G, et al. Factors associated with the development of drug resistance mutations in HIV-1 infected children failing protease inhibitor-based antiretroviral therapy in South Africa. PLoS ONE 2015;107: e0133452. https://doi.org/10.1371/journal.pone. 0133452 .

24. Widiyanti M, Oktavian A, Wibowo HA, et al. Identifikasi genotipe human immunodeficiency virus -1 (HIV-1) pada orang dengan HIV/AIDS (ODHA) di Kota Jayapura Provinsi Papua. Yayapura: Perpustakaan Badan Litbang Kesehatan;2017. 
25. World Health Organization. WHO manual for HIV drug resistance testing using dried blood spot specimens; $3^{\text {rd }}$ ed. Geneva: World Health Organization; 2020.

26. Hoffman J, Van Griensven J, Colebunders R, et al. Role of the CD4 count in HIV management. HIV Ther 2010;4:27-39.

27. Wilson EMP, Sereti I. Immune restoration after antiretroviral therapy: the pitfalls of hasty or incomplete repairs. Immunol Rev 2013;254:34354. DOI: 10.3174/ajnr.A1256.

28. Etta EM, Mavhandu L, Manhaeve C, et al. High level of HIV-1 drug resistance mutations in patients with unsuppressed viral loads in rural northern South Africa. AIDS Res Ther 2017;14:112. DOI: 10.1186/s12981-017-0161-z.

29. Girerd-Genessay I, Baratin D, Ferry T, Chidiac C, Ronin V, Vanhems P. Higher HIV RNA viral load in recent patients with symptomatic acute HIV infection in Lyon University hospitals. PLoS One 2016;11:1-6. DOI: 10.1371/journal.pone.0146978.

30. World Health Organization. Clinical guidelines: antiretroviral therapy. In: Consolidated Guidelines on the use of antiretroviral drugs for treating and preventing HIV infection. Geneva: World Health Organization ;2016. p. 72-190.
31. Memiah P, Shumba C, Etienne-mesubi M, et al. The effect of depressive symptoms and CD4 count on adherence to highly active antiretroviral therapy in sub-Saharan Africa. JIAPAC 2014;13:346-52.DOI: 10.1177/2325957413503368.

32. Chen Y, Chen K, Kalichman SC. Barriers to HIV medication adherence as a function of regimen simplification. Ann Behav Med 2017;176:139-48. DOI: 10.1007/s12160\-016-9827-3.

33. DHHS Panel on Antiretroviral Guidelines for Adults and Adolescents - A Working Group of the Office of AIDS Research Advisory Council (OARAC). Guidelines for the use of antiretroviral agents in adults and aAdolescents. Department of Health and Human Services; 2018.

34. Arguello JR, Noyola DE, Centro C. Prevalence of drug resistance mutations in protease, reverse transcriptase and integrase genes of NorthCentral Mexico HIV isolates. AIDS Res Hum Retroviruses 2017;52:1-29. DOI: 10.1089/AID. 2017.0257.

35. Tanuma J, Hachiya A, Ishigaki K, et al. Impact of CRF01_AE-specific polymorphic mutations G335D and A371V in the connection subdomain of human immunodeficiency virus type 1 (HIV-1) reverse transcriptase (RT) on susceptibility to nucleoside RT inhibitors. Microbes Infect 2010;12:1170-7.DOI: 10.1016/j.micinf.2010.08.003. 